\title{
$125 \mathrm{GeV}$ Higgs and enhanced diphoton signal of a light singlet-like scalar in NMSSM
}

\author{
Marcin Badziak*, Marek Olechowski, Stefan Pokorski \\ Institute of Theoretical Physics, Faculty of Physics, University of Warsaw \\ ul. Hoża 69, PL-00-681 Warsaw, Poland \\ E-mail: mbadziak@fuw.edu.pl, Marek.Olechowski@fuw.edu.pl, \\ Stefan.Pokorskiefuw.edu.pl
}

\begin{abstract}
NMSSM with a light singlet-like scalar and strongly suppressed couplings to $b$ and $\tau$ is investigated. It is shown that in such a scenario the singlet-like scalar to diphoton signal can be larger than for the SM Higgs for a wide range of masses between 60 and $110 \mathrm{GeV}$, in agreement with all the LEP and LHC data. Enhancement of the singlet-like scalar to diphoton signal is correlated with positive correction to the SM-like Higgs mass from mixing between SM-like Higgs and the singlet. It is also shown that the couplings to $b$ and $\tau$ and, in consequence, branching ratios of the SM-like Higgs are anti-correlated with those of the singlet-like scalar. If the singlet-like scalar to diphoton signal is enhanced, the signal strengths of the $125 \mathrm{GeV}$ Higgs in the diphoton and $W W^{*} / Z Z^{*}$ channels are predicted to be smaller than for the SM Higgs.
\end{abstract}

The European Physical Society Conference on High Energy Physics -EPS-HEP2013

18-24 July 2013

Stockholm, Sweden

\footnotetext{
* Speaker.
} 


\section{Introduction}

The measurement of the Higgs mass of about $125 \mathrm{GeV}$ [1,2] stimulated increased interest in extensions of Minimal Supersymmetric Standard Model (MSSM) in which additional positive corrections to the Higgs mass are present allowing for lighter stops relaxing the little hierarchy problem of the MSSM. One of the most attractive and certainly the simplest among those extensions is Next-to-Minimal Supersymmetric Standard Model (NMSSM). In these proceedings we focus on a case in which additional positive correction to the Higgs mass originates from mixing effects in the CP-even scalar sector of NMSSM. We investigate particularly attractive part of parameter space in which the singlet-light scalar is lighter than the discovered Higgs and has suppresed couplings to $b$ and $\tau$. Many (but not all) results presented in these proceedings were originally given in Ref. [3].

\section{CP-even scalar sector in NMSSM}

We begin with a brief summary of the CP-even scalar sector of NMSSM [4]. There are three physical neutral CP-even Higgs fields, $H_{u}, H_{d}, S$ which are the real parts of the excitations around the real vevs, $v_{u} \equiv v \sin \beta, v_{d} \equiv v \cos \beta, v_{s}$ with $v^{2}=v_{u}^{2}+v_{d}^{2} \approx(174 \mathrm{GeV})^{2}$, of the neutral components of doublets $H_{u}, H_{d}$ and the singlet $S$ (we use the same notation for the doublets and the singlet as for the real parts of their neutral components). It is more convenient for us to work in the basis $(\hat{h}, \hat{H}, \hat{s})$, where $\hat{h}=H_{d} \cos \beta+H_{u} \sin \beta, \hat{H}=H_{d} \sin \beta-H_{u} \cos \beta$ and $\hat{s}=S$. The $\hat{h}$ field has exactly the same couplings to the gauge bosons and fermions as the SM Higgs field. The field $\hat{H}$ does not couple to the gauge bosons and its couplings to the up and down fermions are the SM Higgs ones rescaled by $\tan \beta$ and $-\cot \beta$, respectively. The mass eigenstates are denoted as $s, h, H$, with the understanding that $h$ is the SM-like Higgs.

The NMSSM specific part of the superpotential is in general given by ${ }^{1}$

$$
W_{\mathrm{NMSSM}}=\lambda S H_{u} H_{d}+f(S) .
$$

The first term is the source of the effective higgsino mass parameter, $\mu \equiv \lambda v_{s}$, while the second term parametrizes various versions of NMSSM. In the simplest version, known as the scale-invariant NMSSM, $f(S) \equiv \kappa S^{3} / 3$.

We assume also quite general pattern of soft SUSY breaking terms (we follow the conventions used in [4]):

$$
-\mathscr{L}_{\text {soft }} \supset m_{H_{u}}^{2}\left|H_{u}\right|^{2}+m_{H_{d}}^{2}\left|H_{d}\right|^{2}+m_{S}^{2}|S|^{2}+\left(A_{\lambda} \lambda H_{u} H_{d} S+\frac{1}{3} \kappa A_{\kappa} S^{3}+m_{3}^{2} H_{u} H_{d}+\frac{1}{2} m_{S}^{\prime 2} S^{2}+\xi_{S} S+\text { h.c. }\right) .
$$

In the scale-invariant NMSSM, $m_{3}^{2}=m_{S}^{\prime 2}=\xi_{S}=0$.

Let us parametrize the mass matrix of the hatted fields as follows:

$$
\hat{M}^{2}=\left(\begin{array}{ccc}
\hat{M}_{h h}^{2} & \hat{M}_{h H}^{2} & \hat{M}_{h s}^{2} \\
\hat{M}_{h H}^{2} & \hat{M}_{H H}^{2} & \hat{M}_{H s}^{2} \\
\hat{M}_{h s}^{2} & \hat{M}_{H s}^{2} & \hat{M}_{s s}^{2}
\end{array}\right) .
$$

\footnotetext{
${ }^{1}$ Explicit MSSM-like $\mu$-term can also be present in the superpotential but it can always be set to zero by a constant shift of the real component of S.
} 
The off-diagonal terms of the above matrix are given by

$$
\begin{aligned}
& \hat{M}_{h H}^{2}=\frac{1}{2}\left(M_{Z}^{2}-\lambda^{2} v^{2}\right) \sin 4 \beta, \\
& \hat{M}_{h s}^{2}=\lambda v(2 \mu-\Lambda \sin 2 \beta), \\
& \hat{M}_{H s}^{2}=\lambda v \Lambda \cos 2 \beta,
\end{aligned}
$$

where $\Lambda \equiv A_{\lambda}+\left\langle\partial_{S}^{2} f\right\rangle$.

$\hat{M}_{h h}^{2}$ after including radiative corrections, which we parametrize by $\left(\delta m_{h}^{2}\right)^{\mathrm{rad}}$, is given by ${ }^{2}$

$$
\hat{M}_{h h}^{2}=M_{Z}^{2} \cos ^{2}(2 \beta)+\left(\delta m_{h}^{2}\right)^{\mathrm{rad}}+\lambda^{2} v^{2} \sin ^{2}(2 \beta) .
$$

The first two terms in the above equation are the "MSSM" terms, with

$$
\left(\delta m_{h}^{2}\right)^{\mathrm{rad}} \approx \frac{3 g^{2} m_{t}^{4}}{8 \pi^{2} m_{W}^{2}}\left[\ln \left(\frac{M_{\mathrm{SUSY}}^{2}}{m_{t}^{2}}\right)+\frac{X_{t}^{2}}{M_{\mathrm{SUSY}}^{2}}\left(1-\frac{X_{t}^{2}}{12 M_{\mathrm{SUSY}}^{2}}\right)\right],
$$

where $M_{\mathrm{SUSY}} \equiv \sqrt{m_{\tilde{t}_{1}} m_{\tilde{t}_{2}}}\left(m_{\tilde{t}_{i}}\right.$ are the stop masses) and $X_{t} \equiv A_{t}-\mu / \tan \beta$ with $A_{t}$ being SUSY breaking top trilinear coupling at $M_{\text {SUSY }}$. The third term in eq. (2.7) is the new tree-level contribution coming from the $\lambda S H_{u} H_{d}$ coupling.

\subsection{Higgs boson couplings}

Denoting the mass-eigenstates $s, h, H$ by $x=\bar{g}_{x} \hat{h}+\beta_{x}^{(H)} \hat{H}+\beta_{x}^{(s)} \hat{s}$ the couplings (normalized to the corresponding SM values) are given by

$$
\begin{aligned}
& C_{b_{x}}=\bar{g}_{x}+\beta_{x}^{(H)} \tan \beta, \\
& C_{t_{x}}=\bar{g}_{x}-\beta_{x}^{(H)} \cot \beta, \\
& C_{V_{x}}=\bar{g}_{x},
\end{aligned}
$$

where $x$ is $s, h$ or $H$. Note that the couplings to the vector bosons depend only on the $\hat{h}$ components. On the other hand, the couplings to fermions can be modified by mixing with $\hat{H}$.

In the region of moderate and large $\tan \beta$ even small component of $\hat{H}$ in the singlet-dominated Higgs may give a large contribution to the couplings to $b$ quark due to $\tan \beta$ enhancement. On the other hand, the couplings to the up-type quarks are almost the same as those to the gauge bosons, $C_{t_{x}} \approx C_{V_{x}}$. Particularly interesting is the case when $\bar{g}_{s} \beta_{s}^{(H)}<0$ because then $C_{b_{s}} \ll C_{t_{s}}, C_{V_{s}}$ is possible.

It can be shown that $C_{b_{s}}<C_{V_{s}}$ if $\hat{M}_{H s}^{2} \hat{M}_{h s}^{2}<0$ which leads to the following condition for the NMSSM parameters [3]:

$$
\Lambda(\mu \tan \beta-\Lambda) \gtrsim 0
$$

which is satisfied only if $\mu \Lambda>0$.

It is important to stress that strong suppression of the $s b \bar{b}$ coupling does not introduce any new fine-tuning of parameters as long as $\tan \beta$ is large and $\lambda$ is smaller than $\mathscr{O}(0.1)$. The scenario with

\footnotetext{
${ }^{2}$ The formulea for the diagonal entries of $\hat{M}^{2}$ are not relevant for our present discussion and can be found in Ref. [3].
} 
large $\lambda \gtrsim 0.6$ and small $\tan \beta$ that lead to substantial tree-level contribution to $m_{h}$ requires at least few-percent fine-tuning to avoid negative eigenvalues of the Higgs mass matrix [3].

In the regime $C_{b_{s}} \ll C_{t_{s}}, C_{V_{s}}$, the (otherwise dominating) $s$ branching ratios to $b \bar{b}$ and $\tau \bar{\tau}$ are strongly suppressed and $s$ decays mainly to $g g$ and $c \bar{c}$. This has many important implications which we discuss in following sections.

\section{Correction to the Higgs mass from mixing}

In NMSSM, in addition to the correction coming from the $\lambda$ coupling, there exist also correction from mixing between scalars, $\Delta_{\text {mix }}$, which we parametrize as follows:

$$
m_{h}=\hat{M}_{h h}+\Delta_{\text {mix }} .
$$

Mixing with $\hat{s}$ gives positive (negative) contribution to $\Delta_{\text {mix }}$ if the singlet-dominated scalar is lighter (heavier) then the SM-like Higgs, while the contribution to $\Delta_{\text {mix }}$ from mixing with $\hat{H}$ is negative and gets smaller in magnitude for larger values of $m_{H}$. Therefore, in order to calculate the maximal allowed value of $\Delta_{\text {mix }}$ it is enough to consider a case in which $H$ is decoupled. In such a case $\Delta_{\text {mix }}$ is given by

$$
\Delta_{\text {mix }}=m_{h}-\sqrt{m_{h}^{2}-\bar{g}_{s}^{2}\left(m_{h}^{2}-m_{s}^{2}\right)} \approx \frac{\bar{g}_{s}^{2}}{2}\left(m_{h}-\frac{m_{s}^{2}}{m_{h}}\right)+\mathscr{O}\left(\bar{g}_{s}^{4}\right),
$$

where in the last, approximate equality we used the expansion in $\bar{g}_{s}^{2} \ll 1$. It is clear from the above formula that a substantial correction to the Higgs mass from the mixing is possible only for not too small couplings of the singlet-like state to the $Z$ boson and that $m_{s} \ll m_{h}$ is preferred. However, LEP has provided rather strong constraints on the states with masses below $\mathscr{O}(110) \mathrm{GeV}$ that couple to the $Z$ boson because such states could be copiously produced in the process $e^{+} e^{-} \rightarrow s Z$.

For those LEP searches that rely on $b$ and $\tau$ tagging [5], constraints on $\bar{g}_{s}^{2}$ depend on the $s$ branching ratios and the LEP experiments provide constraints on the quantity $\xi^{2}$ defined as:

$$
\xi_{b \bar{b}}^{2} \equiv \bar{g}_{s}^{2} \times \frac{\mathrm{BR}(s \rightarrow b \bar{b})}{\mathrm{BR}^{\mathrm{SM}}(h \rightarrow b \bar{b})} .
$$

The LEP constraints on $\xi_{b \bar{b}}^{2}$ are reproduced by the red line in the left panel of Figure 1. Assuming $C_{b_{s}} \approx C_{t_{s}}, C_{V_{s}} \approx \bar{g}_{s}$, i.e. neglecting the mixing with $\hat{H}$, the branching ratios of $s$ are the same as for the SM Higgs. Therefore, the limits on $\xi_{b \bar{b}}^{2}$ depicted by the red line in Figure 1 are, in fact, also the limits on $\bar{g}_{s}^{2}$. Using eq. (3.2) we can translate the constraints on $\bar{g}_{s}^{2}$ into limits for the maximal allowed correction from the mixing, $\Delta_{\operatorname{mix}}^{\max }$, as a function of $m_{s}$. These are presented in the right panel of Figure 1. Notice that in this case $\Delta_{\text {mix }}$ can reach about $6 \mathrm{GeV}$ in a few-GeV interval for $m_{s}$ around $95 \mathrm{GeV}$, where the LEP experiments observed the $2 \sigma$ excess in the $b \bar{b}$ channel. However, for $m_{s} \lesssim 90 \mathrm{GeV}$ the allowed value of $\Delta_{\text {mix }}^{\max }$ drops down very rapidly to very small values.

The situation changes if the $s b \bar{b}$ coupling is strongly suppressed because in such a case the constraints from the $b$-tagged LEP searches become meaningless. The main constraint on such a scenario is provided by the flavour independent search for a Higgs decaying into two jets at LEP [6]. Those searches give constraints on a quantity $\xi_{j j}^{2}$ defined as:

$$
\xi_{j j}^{2} \equiv \bar{g}_{s}^{2} \times \mathrm{BR}(s \rightarrow j j)
$$



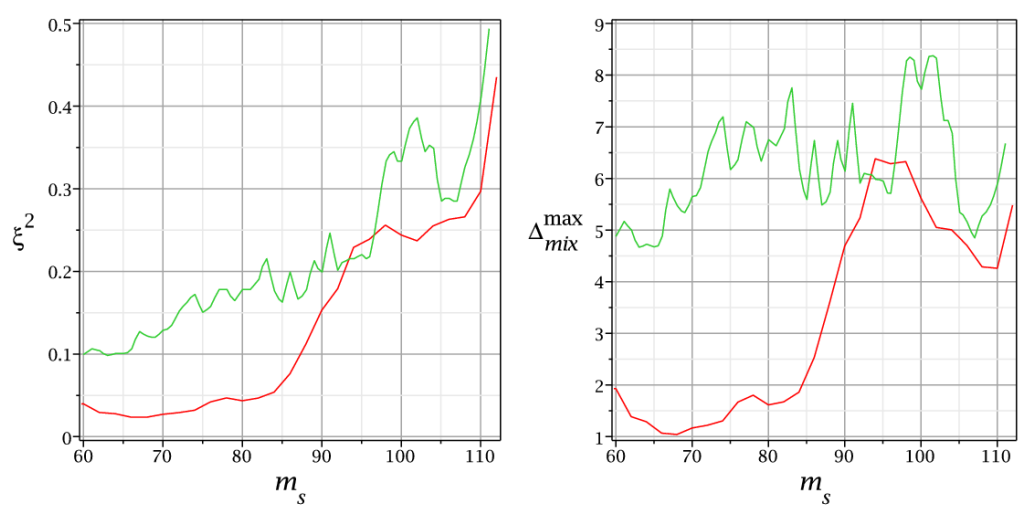

Figure 1: Left: The $95 \%$ CL upper bounds on $\xi_{b \bar{b}}^{2}$ (the red line) or $\xi_{j j}^{2}$ (the green line). The red line was obtained using the observed limits presented in the column (a) of Table 14 in Ref. [5], while the green line corresponds to Figure 2 of [6]. Right: The LEP limits translated to the upper limits on $\Delta_{\operatorname{mix}}$ using eq. 3.2 assuming $\xi_{b \bar{b}}^{2}=\bar{g}_{s}^{2}$ and $\xi_{j j}^{2}=\bar{g}_{s}^{2}$ for the red and green line, respectively.

which are reproduced by the green line in the left panel of Figure 1. Noting that for suppressed $s b \bar{b}$ and $s \tau \bar{\tau}$ couplings, $\mathrm{BR}(s \rightarrow j j) \approx 1$ so $\xi_{j j}^{2} \approx \bar{g}_{s}^{2}$, it becomes clear that these constraints are substantially weaker and allow for values of $\bar{g}_{s}^{2}$ above 0.3 for $m_{s}$ around $100 \mathrm{GeV}$ and the limit rather slowly improves as $m_{s}$ goes down, as seen from the left panel of Figure 1. In consequence, the constraints on $\Delta_{\text {mix }}^{\max }$ are also weaker. As can be seen from the right panel of Figure 1, when $s \rightarrow b \bar{b}$ decays are suppressed $\Delta_{\text {mix }}$ above $5 \mathrm{GeV}$ is viable for a large range of $m_{s}$ with a maximum of about $8 \mathrm{GeV}$ for $m_{s}$ around $100 \mathrm{GeV}$.

\subsection{Anti-correlation between the $s$ and $h$ couplings}

It is very interesting to note that the $s b \bar{b}$ coupling is anti-correlated with the $h b \bar{b}$ coupling. The proof of this fact is given in Ref. [3]. This implies that for suppressed $s b \bar{b}$ coupling, $R_{V V}^{(h)}<1-\bar{g}_{s}^{2}$ (where $V=W$ or $Z$, and $R_{V V}^{(h)}$ is the cross-section times branching ratio normalized to the SM value). In order to study quantitatively this feature of the scenario we performed a numerical scan over the NMSSM parameter space for various values of $m_{s}$ and $m_{H}$ while keeping fixed $m_{h}=125$ $\mathrm{GeV}$. Details of the scan can be found in Ref. [3] and the results are presented in Figures 2 and 3. In Figure 2 scatter plots of $\Delta_{\text {mix }}$ versus $m_{s}$ are presented. The LEP constraints discussed before have been taken into account. It can be seen from the left panel that the biggest $\Delta_{\text {mix }}$ corresponds to small values of $R_{V V}^{(h)}$ (note that for large $\tan \beta R_{\gamma \gamma}^{(h)} \approx R_{V V}^{(h)}$ ), in tension with the LHC Higgs data. If one demands consistency with the LHC Higgs data at 95\% CL, $\Delta_{\operatorname{mix}}^{\max }$ is between about 5 and 7 $\mathrm{GeV}$ for $m_{s}$ between $m_{h} / 2$ and $105 \mathrm{GeV}$, as can be seen from the right panel of Figure 2.

\section{Enhanced $s \rightarrow \gamma \gamma$}

In the scenario with strongly supressed $s b \bar{b}$ and $s \tau \bar{\tau}$ couplings the total decay width of $s$ is strongly reduced so all the $s$ branching ratios, except those for the $s$ decays to the down-type fermions, are strongly enhanced. Particularly interesting, from the viewpoint of prospects for dis- 

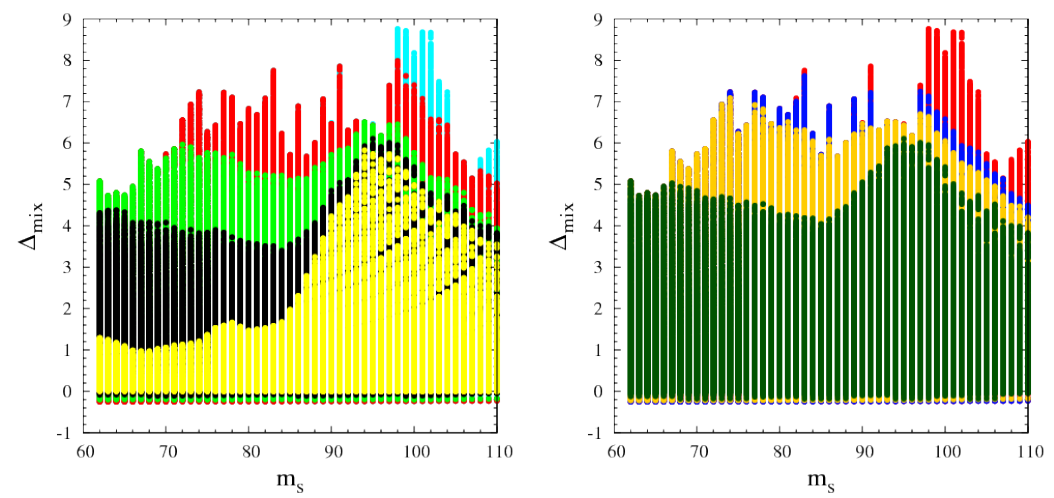

Figure 2: Results of the numerical scan presented in the $\Delta_{\mathrm{mix}}-m_{s}$ plane after taking into account the LEP constraints. Left: The blue points are characterised by $R_{V V}^{(h)}<0.5$ while for the red, green, black and yellow points $R_{V V}^{(h)}$ is larger than $0.5,0.7,0.8$ and 1, respectively. The points with larger values of $R_{V V}^{(h)}$ are overlaid on the points with smaller $R_{V V}^{(h)}$. Right: Color denotes $p$-value based on the $125 \mathrm{GeV}$ Higgs data updated for this conference. Green, yellow, blue, red points correspond to $p$-value above 0.32 , between 0.32 and 0.05 , between 0.05 and 0.01 , below 0.01 , respectively.
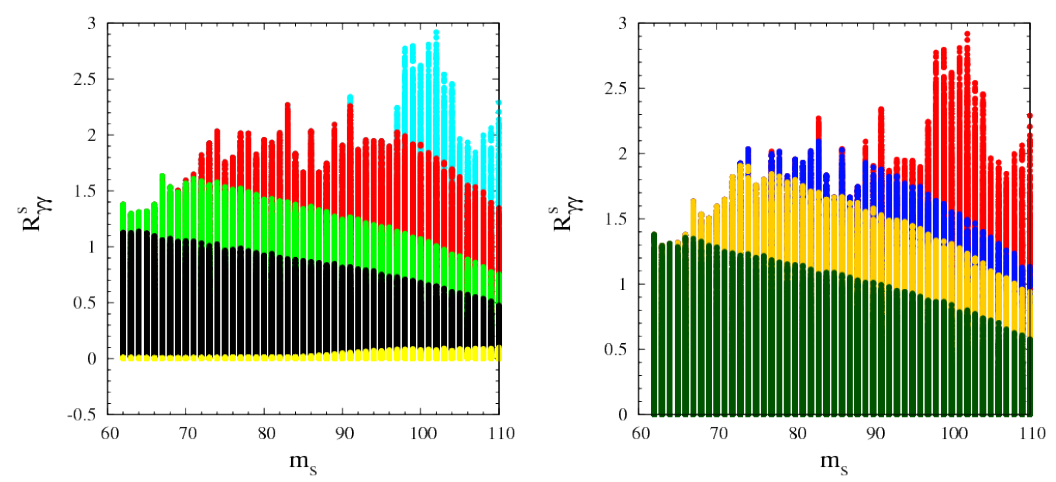

Figure 3: The same as in Figure 2 but in the $R_{\gamma \gamma}^{(s)}-m_{s}$ plane.

covery of $s$, is the $\gamma \gamma$ final state. Even though the production cross-section is suppressed by $\bar{g}_{s}^{2}$, $\mathrm{BR}(h \rightarrow \gamma \gamma)$ can be enhanced by more than a factor of ten resulting in the signal strength $R_{\gamma \gamma}^{(s)}>1$.

It can be seen from Figure 3 that after taking into account the LEP constraints $R_{\gamma \gamma}^{(s)}$ can be up to about three. However, it is also seen from the Figure that very large $R_{\gamma \gamma}^{(s)}$ typically correspond to small $R_{V V}^{(s)}$ and is incompatible with the LHC data for the $125 \mathrm{GeV}$ Higgs. Nevertheless, even after taking into account these constraints $R_{\gamma \gamma}^{(s)}>1$ can be obtained for a wide range of $m_{s}$ between about 60 and $110 \mathrm{GeV}$, and $R_{\gamma \gamma}^{(s)}$ can reach two for $m_{s}$ around $70 \mathrm{GeV}$.

\section{Conclusions}

We investigated NMSSM with a light singlet-like scalar with suppressed couplings to $b$ and $\tau$. 
In this scenario the discovered Higgs boson is next to the lightest one and its mass can get positive correction of about 5-7 GeV from mixing with the singlet for a wide range of $m_{s}$ between 60 and $110 \mathrm{GeV}$. Thus, stop masses can be substantially smaller than in the MSSM.

Characteristic signature of this scenario is enhanced $s \rightarrow \gamma \gamma$ signal which can be up to three times larger than for the SM Higgs in agreement with all the LEP data. Even though direct Higgs searches in the diphoton channel have not been performed for masses below $110 \mathrm{GeV}$ at the LHC so far, the LHC results already constrain this scenario because the $125 \mathrm{GeV}$ Higgs production cross-section and branching ratios to gauge bosons are always smaller than in the SM. This is because mixing of $\hat{h}$ with the singlet reduces the Higgs production cross-section, while suppression of the $s b \bar{b}$ coupling always imply enhancement of the $h b \bar{b}$ coupling. We found that the NMSSM points predicting the largest enhancement of the $s \rightarrow \gamma \gamma$ signal are ruled out by the $125 \mathrm{GeV}$ Higgs measurements but the signal strength two times larger than for the SM Higgs is still possible. Therefore, we encourage the LHC collaborations to extend direct Higgs searches in the diphoton channel to masses between 60 and $110 \mathrm{GeV}$.

Suppression of the $s b \bar{b}$ coupling is naturally present in NMSSM with moderate and large $\tan \beta$ and small values of $\lambda$. Such suppression is also possible for small $\tan \beta$ and large $\lambda$ but at the cost of additional fine-tuning of the NMSSM parameters.

\section{Acknowledgments}

This work is a part of the "Implications of the Higgs boson discovery on supersymmetric extensions of the Standard Model" project funded within the HOMING PLUS programme of the Foundation for Polish Science. MO and SP have been supported by National Science Centre under research grants DEC-2011/01/M/ST2/02466, DEC-2012/04/A/ST2/00099, DEC-2012/05/B/ST2/02597. MB has been partially supported by the MNiSW grant IP2012 030272 and the Foundation for Polish Science through its programme START.

\section{References}

[1] G. Aad et al. [ATLAS Collaboration], Observation of a new particle in the search for the Standard Model Higgs boson with the ATLAS detector at the LHC, Phys. Lett. B 716 (2012) 1 [arXiv:1207.7214 [hep-ex]].

[2] S. Chatrchyan et al. [CMS Collaboration], Observation of a new boson at a mass of $125 \mathrm{GeV}$ with the CMS experiment at the LHC, Phys. Lett. B 716 (2012) 30 [arXiv:1207.7235 [hep-ex]].

[3] M. Badziak, M. Olechowski and S. Pokorski, New Regions in the NMSSM with a $125 \mathrm{GeV} \mathrm{Higgs,}$ JHEP 1306 (2013) 043 [arXiv:1304.5437 [hep-ph]].

[4] U. Ellwanger, C. Hugonie and A. M. Teixeira, The Next-to-Minimal Supersymmetric Standard Model, Phys. Rept. 496 (2010) 1 [arXiv:0910.1785 [hep-ph]].

[5] S. Schael et al. [ALEPH and DELPHI and L3 and OPAL and LEP Working Group for Higgs Boson Searches Collaborations], Search for neutral MSSM Higgs bosons at LEP, Eur. Phys. J. C 47 (2006) 547 [hep-ex/0602042].

[6] LEP Higgs Working Group for Higgs boson searches collaboration, Flavor independent search for hadronically decaying neutral Higgs bosons at LEP, hep-ex/0107034. 